\title{
Estrategias para diseño de vivienda social en Cuenca - Ecuador, mediante un análisis cuantitativo de patrones sociales, económicos, habitacionales y constructivos
}

Strategies for the design of social housing in Cuenca - Ecuador, through a quantitative analysis of social, economic, housing and construction

patterns

Álvaro Rafael Toledo Toledo. ${ }^{1}$, Jorge Fernando Toledo Toledo. ${ }^{2} \&$ Marco Avila
Calle. ${ }^{3}$

Recibido: 25-02-2021 / Revisado: 03-03-2021 /Aceptado: 12-03-2021/ Publicado: 05-04-2021

\begin{abstract}
DOI: https://doi.org/10.33262/cienciadigital.v5i2.1595

The housing projects in the city of Cuenca - Ecuador, only satisfy the needs of the housing deficit in the middle and high sectors, because the cost is too high for the lower social class, due to the lack of regulations and specific studies to determine the optimal urban soils, construction systems and design strategies to promote low-cost housing. The research project, in addition to considering the social conditions of habitability of the lowincome population, to establish the spatial requirements, as well as the most appropriate location for the implementation of the project, allows to establish design strategies to achieve a sustainable and affordable proposal to the social group of study, supported by quantitative and qualitative methods. At the end of the research, a set of guidelines and recommendations for architectural design and construction will be obtained for housing projects for the low-income population categorized in the social stratum C-and D in the city of Cuenca, and could be applicable in any city in Ecuador or Latin America that has similar conditions to the study universe. The impact of the research will be evidenced in the potential public policies of habitability generated through regulations or ordinances

\footnotetext{
${ }^{1}$ Universidad Católica de Cuenca, Maestría en Construcciones con mención en Administración de la Construcción Sustentable, Cuenca, Ecuador. alvarorafa78@gmail.com. ORCID https://orcid.org/00000003-3862-785

${ }^{2}$ Universidad Católica de Cuenca, Facultad de Arquitectura. Cuenca, Ecuador. jftoledot@ucacue.edu.ec. ORCID https://orcid.org/0000-0003-0007-7510

3 Universidad Católica de Cuenca, Facultad de Arquitectura. Cuenca, Ecuador. mavila@ucacue.edu.ec. ORCID https://orcid.org/0000-0002-2134-1432
} 
in the city of Cuenca - Ecuador, through the design and construction recommendations obtained in the research.

Keywords: social housing, habitability, design strategies.

\section{Resumen}

Los proyectos habitacionales en la ciudad de Cuenca - Ecuador, solo satisfacen las necesidades del déficit de vivienda en los sectores medio y alto, pues el costo es muy elevado para que pueda acceder la clase social baja, debido a la falta de normativas y estudios específicos para determinar los suelos urbanos óptimos, sistemas constructivos y estrategias de diseño que permitan propiciar la vivienda a bajo costo. El proyecto de investigación a más de considerar las condiciones sociales de habitabilidad que presenta la población de bajos recursos económicos, para establecer los requerimientos espaciales, así como la ubicación más apropiada para la implementación del proyecto, permite establecer estrategias de diseño para conseguir una propuesta sustentable y asequible al grupo social de estudio, apoyados en métodos cuantitativos y cualitativos. Al final de la investigación se obtendrán un conjunto de lineamientos y recomendaciones de diseño arquitectónico y construcción para proyectos habitacionales destinados a la población de bajos recursos económicos categorizados en el estrato social C- y D en la ciudad de Cuenca, pudiendo ser aplicable en cualquier ciudad del Ecuador o Latinoamérica que tenga las condiciones similares al universo de estudio. El impacto de la investigación se evidenciará en las potenciales políticas públicas de habitabilidad que se generen mediante normativas $\mathrm{u}$ ordenanzas en la ciudad de Cuenca - Ecuador, a través de las recomendaciones de diseño y construcción obtenidas en la investigación.

Palabras claves: Vivienda social, habitabilidad, estrategias de diseño.

\section{Introducción}

La escasez cualitativa y cuantitativa de conjuntos habitacionales para familias de bajos recursos son alarmantes, la limitación a servicios básicos afecta a un sinnúmero de hogares de la región: El 21\% no disponen de instalaciones hidrosanitarias y electricidad; $12 \%$ de las viviendas están fabricadas de materiales de construcción inadecuados, otro porcentaje que fluctúa en el $6 \%$ disponen de un suelo de tierra o viven en condiciones de aglomeración, estas características perjudican directamente la salud de los habitantes ("Estudio del BID: América Latina y el Caribe encaran creciente déficit de vivienda | IADB," n.d.). En el curso de esta época más del 90\% de la ciudadanía hará su vida en las ciudades, situación que genera un aumento descontrolado en su extensión, con el coligado aumento de los precios de urbanización y con listas grandes de economías de escala. A lo indicado en líneas anteriores, hay que agregar la discriminación socio-espacial (Ghetto), con grandes inequidades en materia de acceso a servicios y bienes urbanos (Inmobiliario 
2005) lo que complica a ciertas secciones poblacionales la obtención de una vivienda. En la capital del Azuay el incremento de la localidad en el año 2001 fue de 612.565 habitantes y al año 2010 fue 702.893 habitantes, es decir un 14.7\% de aumento según estos datos, la carencia de lugares habitacionales es de 35.000 a 45.000 (Muñoz, Patricia, Ochoa, \& Maritza, 2011), teniendo afectados de forma directa el estrato social C- y D.

Las variaciones económicas encaminadas al mercado y al sector privado que se han instituido en América Latina y el Caribe en los últimos años han traído consigo notables redefiniciones en las políticas de vivienda para hogares de menores ingresos. Esas políticas han demostrado que pueden expandir en forma considerable la acogida de viviendas sociales y acortar las carencias habitacionales. Sin embargo, también han ofrecido escaso cuidado al déficit cualitativo de vivienda, a los suelos para unidades de interés social, y al mercado secundarios (Franco, 2013). Ya en el entorno nacional, el estado ecuatoriano a través del Plan Nacional del Buen Vivir (en el objetivo 3), propone entre los derechos para perfeccionar la calidad de vida, incluir (art.14, un hábitat seguro y saludable, a una vivienda digna con independencia de la situación social y económica (Held, 2000). Entendiéndose que la clase social tipo C - y D es aquella que sus entradas económicas no van más allá del salario básico unificado (SBU) mensual por ello este análisis permitirá precisar las recomendaciones de diseño, para establecer donde es ventajoso plantear proyectos para la vivienda social, que espacios y que estrategias debe tener la propuesta, mejorando la calidad de vida y optimizando el uso de suelo urbano en la ciudad; favoreciéndose de esta información la entidad municipal, arquitectos, ingenieros y sobre todo los estratos sociales menos protegidos de Cuenca.

Dado el crecimiento de la población en el Ecuador y por ende en la provincia del Azuay, este problema no es diferente en el país, así como en la provincia en la que se realiza el estudio. En el año 2001, la población era de 612.565 habitantes; en 2010, incrementó a 702.893 , teniendo como tal un aumento del 14,7\% en la última década; por lo tanto, es lógico que la demanda de vivienda también se ha elevado. Según el Instituto Nacional de Estadísticas y Censos (INEC, 2010), en el sector de la construcción, los permisos emitidos por los Gobiernos Autónomos Descentralizados han crecido a una tasa promedio anual del $12 \%$ al 14\% desde 2009, según estas estadísticas, existe un déficit de 35.000 a 45.000 soluciones habitacionales en la ciudad de Cuenca.

La Empresa Municipal de Vivienda (EMUVI), tuvo previsto construir viviendas con precios asequibles en el año 2011, las mismas fluctuaban entre 15.000, 25.000 y 30.000 dólares estadounidenses e incluían 3 habitaciones: sala, cocina y comedor; pero hasta ahora, este proyecto no se ha plasmado. Sin embargo, se han dado programas municipales de dotación de viviendas a través de comunidades cerradas; no obstante, en el proceso de planificación, promoción y venta, de estos proyectos se han visto beneficiados las clases media y alta, porque la clase baja no puede financiarse por los altos costos que representan tanto el sitio como las edificaciones. 
El problema se ha agravado cuando el mercado inmobiliario ha experimentado un crecimiento sustancial en los últimos años debido a la demanda de construcciones por parte de inversionistas locales, nacionales y extranjeros. Por lo tanto, el costo por metro cuadrado de terreno en áreas urbanas y rurales es de alrededor del $300 \%$ del precio original; en tanto que la construcción se ha incrementado un 50\%, motivo que hace imposible a cierta población la adquisición de una casa.

Personeros de la Empresa Municipal de Vivienda (EMUVI) y del Ministerio de Desarrollo Urbano y Vivienda (MIDUVI), gerente y subsecretario respectivamente, revelan que la obtención de terrenos adecuados para construcciones es el principal problema para los proyectos de vivienda, porque los trabajos de redes de agua potable y alcantarillado no son una tarea fácil, ya que implica que los cabildos municipales inviertan en realizar estas obras aumentando así el costo final de los proyectos de vivienda de interés social. Debido a esta problemática, entidades financieras como Mutualista Azuay han emprendido en propuestas de vivienda social, pero han tropezado con algunos problemas. El gerente de la entidad financiera señaló que existe una proyección municipal que no es compatible con el valor del suelo; por lo tanto, esperan que las autoridades tomen en cuenta estas circunstancias, y que se apruebe construir edificaciones con mayor densidad, y facilitar servicios hidrosanitarios a predios urbanos con características físicas y geológicas para construcciones arquitectónicas, lo que daría paso a realizar obras de orden social, puesto que las leyes y regulaciones urbanas vigentes no permiten aprovechar al máximo la potencialidad del uso del suelo urbano.

Actualmente, a medida que los diseñadores enfrentan desafíos para proporcionar viviendas cómodas y costos adecuados para los sectores de bajos ingresos, la demanda de viviendas asequibles en los países en desarrollo de América Latina ha atraído gran atención. En Cuenca, el problema se va agravando ya que la normativa limita la densificación de áreas urbanas con características adecuadas para construir edificios en altura, este escenario desata un crecimiento desmedido e incontrolable a lo largo de ejes urbanos no planificados, lo que provoca que el Municipio de Cuenca no pueda afrontar el equipamiento e infraestructura básica, esto provocaría un incremento del valor en el bien a ser edificado.

Para determinar el espacio habitacional para grupos de bajos ingresos, se dispone de datos del INEC, pero los mismos no pueden definir los requerimientos de espacio para un proyecto, por lo que se decidió realizar una encuesta para tal fin.

En Ecuador, el gobierno propuso mejorar la calidad de vida de la población a través de espacios inclusivos, equitativos y dignos, a través del Plan Nacional "Toda una vida", por lo que se vuelve imperioso establecer un proyecto de viviendas confortables para los sectores de bajos recursos económicos.

La investigación tiene como objetivo proporcionar modelos teóricos derivados de una investigación cuantitativa y cualitativa, a partir de lo cual se analizan las variables de 
carácter (cuantitativas), que tienen como objetivo, categorizar los ámbitos: sociales, económicos, habitacionales y constructivos de los proyectos de vivienda, para el estrato social C - D en la ciudad de Cuenca.

\section{Metodologia}

El tipo de investigación define el alcance del objetivo, según la naturaleza de la información recolectada para dar respuesta a la pregunta de investigación, en el presente caso es de enfoque cuantitativo, porque la búsqueda de datos se basa principalmente en su cuantificación y cálculo.

La investigación que se plantea corresponde a un enfoque compuesto porque en el estudio los datos se cuantifican y a la vez se detallan y explican cualitativamente la realidad social y económica de las personas de escasos recursos. En consecuencia, la investigación se enmarca en el nivel Descriptivo - Explicativo, porque se concentra en estudiar las causas o los efectos de un determinado hecho por medio de la prueba de hipótesis.

El diseño de la investigación se define con base al procedimiento, el diseño se refiere en DÓNDE y CUÁNDO se recopila la información, así como la amplitud de la información recopilada, de modo que se pueda dar respuesta a la pregunta de investigación de la forma más idónea posible (Hernández M., 2012)

\section{EL DÓNDE}

Las fuentes de información para establecer el objetivo de las características sociales y económicas, de la población de bajos recursos económicos de la ciudad de Cuenca, mismas que determinan el poder adquisitivo y las necesidades espaciales para la vivienda; estas son vivas y la información se recoge en su ambiente natural por medio de encuestas, por tanto, el diseño es de campo.

\section{EL CUÁNDO}

La toma de información corresponde a una situación actual tanto la de campo como la documentada, por tanto, es contemporánea.

\section{POBLACIÓN}

Para la determinación de las características sociales y económicas se realizan encuestas partiendo de considerar que en la ciudad de Cuenca hay una población de 505.585 habitantes (INEC, 2010), los resultados obtenidos en el cálculo de pobreza y desigualdad a partir de la Encuesta Nacional de Empleo, Desempleo y Subempleo (ENEMDU)1 del mes de marzo 2014, (INEC, 2014) la ciudad que registra el menor nivel de pobreza es Cuenca (4.67\%), donde existe una disminución en el periodo de 4,5 puntos porcentuales; esta variación es estadísticamente significativa. Esto implica que alrededor de 23.611 habitantes viven en la pobreza; se determina que el grupo familiar tiene en promedio 4 
miembros (INEC, 2010), correspondiendo a 5902 hogares de escasos recursos que constituye en la población de análisis.

\section{MUESTRA}

Para definir el número total de la muestra a encuestar se aplica la siguiente ecuación:

$$
n=\frac{N \times Z^{2} \times p \times q}{d x(N-1)+Z^{2} \times p}
$$

donde:

$\boldsymbol{n}=$ Número de muestra

$N=$ Total de la población

$\mathbf{Z}=$ Nivel de confianza $=1.645$

$p=$ Proporción esperada $=50 \%=0.50$

$q=(1-p)=0.50$

$\boldsymbol{d}=$ La prevención del $5 \%=0.05$

Aplicando la ecuación para identificar el tamaño de la muestra, se tiene que el número de encuestados deberían ser 280 hogares, sin embargo, por la disponibilidad se aplicó el instrumento a un total de 450 hogares.

Tabla 01. Variables de la investigación

\begin{tabular}{cc}
\hline Variable independiente & $\begin{array}{c}\text { Vivienda social } \\
\text { Ámbito económico }\end{array}$ \\
Variable dependiente & Ámbito social \\
& Ámbito habitacional \\
& Ámbito constructivo \\
\hline
\end{tabular}

Elaboración: Toledo A.

\section{TÉCNICAS}

Para el proceso, se hace uso de la encuesta y el análisis documental a través del fichaje.

\section{INSTRUMENTOS}

Se utiliza la guía de análisis de contenido para recopilar información acerca de los indicadores estadísticos del INEC, al igual que las preguntas de encuesta para compilar datos de fuentes primarias que en este caso es la población de escasos recursos económicos, también la ficha de observación juega un papel importante que con este instrumento se registra información del análisis de sitio, y como es lógico no se puede dejar de lado los tecnología digital como cámaras, grabadoras, filmadoras, celulares, los mismos que permiten registrar datos en tiempo real, garantizando la autenticidad de la muestra a ser estudiada. 


\section{TÉCNICAS DE PROCESAMIENTO}

El presente proyecto consta de dos instancias: La primera inicia con la recolección de datos en las fuentes de información disponibles como es la oficina de EMUVI-EP, Alcaldía de Cuenca, el Ministerio de Desarrollo Urbano y Vivienda MIDUVI y la ejecución de 450 encuestas, mediante lo cual se identifican los requerimientos de clase social C - y D. Esta información da paso al estudio de características cuantitativas para su análisis y evaluación.

Partiendo del diagnóstico e información obtenida de las encuestas y a través de la aplicación de herramientas estadísticas, se procesa la información para obtener datos que permitan definir los lineamientos de diseño para propuestas de vivienda de interés social que contribuyan a satisfacer las necesidades, costos y expectativas de la población de bajos ingresos económicos.

\section{Resultados}

La encuesta se dividió en tres secciones: ámbito social, ámbito económico y ámbito habitacional - constructivo.

De los resultados analizados, se puede establecer que los hogares están formados por gente joven hasta 50 años, solo en un 19\% de los hogares existen adultos mayores.

La vivienda está habitada en su mayoría por una sola familia, con 2 y 3 cargas familiares, pero el grupo familiar principal de las encuestas se puede determinar que está compuesta de 4 miembros en su mayoría como se puede observar en los gráficos 1 y 2 :

\section{CARGAS FAMILIARES}

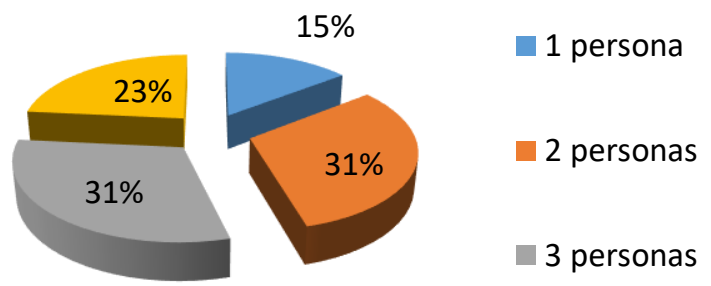

Gráfico 1. Número de cargas familiares. Fuente: Elaboración propia. 


\section{COMPOSICIÓN DEL NUCLEO \\ FAMILIAR}

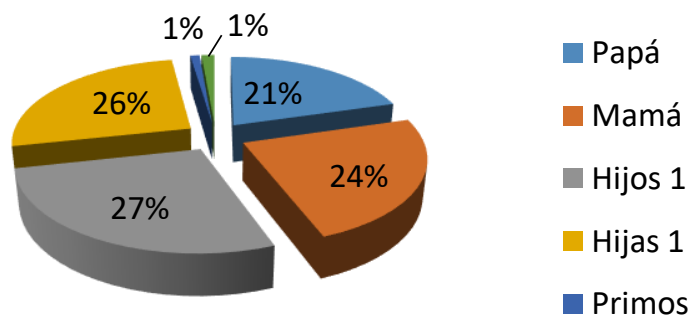

Gráfico 2. Composición del núcleo familiar. Fuente: Elaboración propia.

Tanto padre y madre trabajan para aportar económicamente al hogar, igual se puede deducir también que los hijos cuando alcanzan la mayoría de edad empiezan a trabajar para aportar al hogar, pues se puede verificar que los porcentajes de hijos e hijas que trabajan tienen correspondencia casi directa con los porcentajes de edad a partir de los 20 años.

La discapacidad física se presenta en un $23 \%$ de los hogares, tal como lo demuestra el gráfico 3, porcentaje representativo que debe tomarse en cuenta al momento de diseñar cualquier elemento arquitectónico.

\section{PERSONAS CON DISCAPACIDADES}

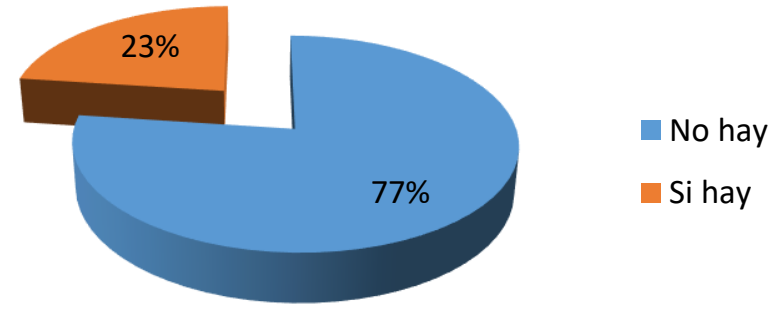

Gráfico 3. Porcentaje de personas con discapacidades. Fuente: Elaboración propia

Los hijos pequeños en un $65 \%$ se quedan en la casa y en un $21 \%$ con familiares, tan solo un $5 \%$ asisten a guarderías, esto nos permite establecer que una muy buena ayuda a estas familias sería proponer en el espacio de participación municipal la construcción de una guardería municipal para el control y cuidado de los niños de este grupo social. En el ámbito económico los ingresos de una persona está entre 300 a 400 dólares en el 35\% de los hogares y más de 400 dólares en el 36\% de estos, siendo estos ingresos en su mayoría por sueldo mensual, mientras que sus gastos están repartidos de forma similar según los porcentajes del cuadro respectivo, pero en todo caso se puede decir que alcanza hasta 400 dólares en el 27\% y más de 400 dólares en el 24\% de los hogares, al ser dos personas las 
que aportan en el mayor porcentaje en estos hogares, se puede deducir que tienen capacidad económica para invertir en la adquisición de un apartamento, como lo corrobora el $29 \%$ de familias que dicen poder invertir hasta 20000 dólares, tal como lo demuestran los gráficos 4,5 y 6 .

\section{VALOR DE INGRESOS EN EL HOGAR: (DE UNA SOLA} PERSONA)

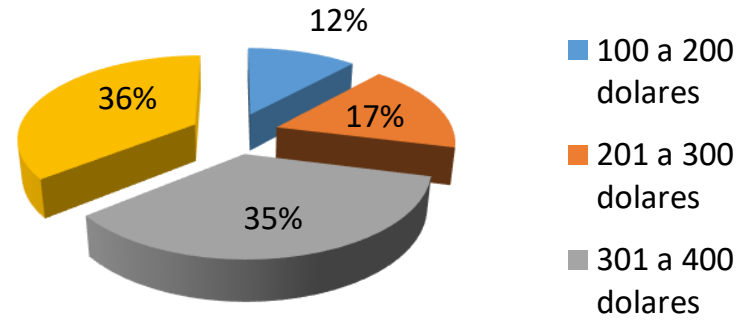

Gráfico 4. Ingresos económicos de una sola persona del hogar. Fuente: Elaboración propia

\section{VALOR DE EGRESOS EN EL HOGAR}

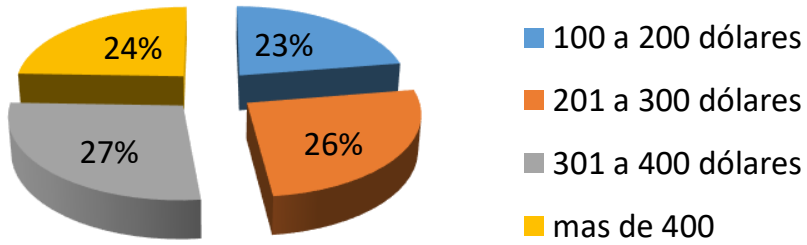

Gráfico 5. Egresos económicos totales del hogar. Fuente: Elaboración propia

MONTO QUE PUEDEN INVERTIR PARA ADQUIRIR APARTAMENTO

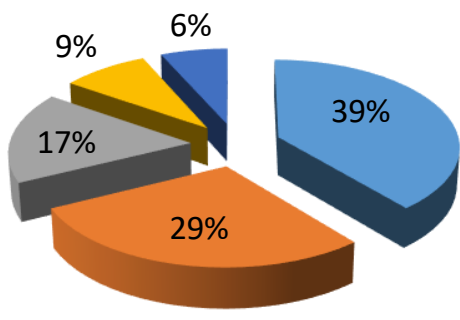

$$
\begin{aligned}
& \text { No puede } \\
& 20 \text { mil dólares } \\
& 25 \text { mil dólares } \\
& 30 \text { mil dólares } \\
& -35 \text { mil o más }
\end{aligned}
$$

Gráfico 6. Monto que pueden invertir para adquirir un departamento. Fuente: Elaboración propia 
El medio de transporte propio está en un $28 \%$ y el público en un $71 \%$ lo que permite establecer que lo planteado en la norma municipal para vivienda de interés social de una plaza de estacionamiento por cada 3 unidades de vivienda es apropiada.

De los resultados del ámbito habitacional y constructivo, se puede establecer que si bien un $35 \%$ de hogares viven arrendando existe un $19 \%$ de viviendas que acogen a dos familias, un $4 \%$ a tres familias y un $2 \%$ a más de cuatro familias lo que permite deducir que el porcentaje de familias que no tiene una vivienda asciende al $65 \%$ por lo que el déficit de vivienda es considerable para este grupo social. Ver gráfico 7.

\section{LA VIVIENDA ES}

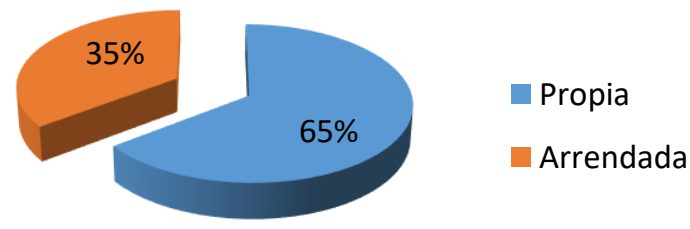

\section{Gráfico 7. La vivienda es propia o arrendada. Fuente: Elaboración propia}

En la vivienda predomina el enlucido y pintado de sus paredes, la madera en sus pisos, el cemento y cerámica en el resto de la muestra, el baño social dispone el $44 \%$ de los hogares encuestados, el $90 \%$ disponen de un baño completo para el grupo familiar, esto se lo puede verificar en los gráficos del 8 y 9.

\section{ACABADO QUE PREDOMINA EN LA PARED DE LA VIVIENDA}

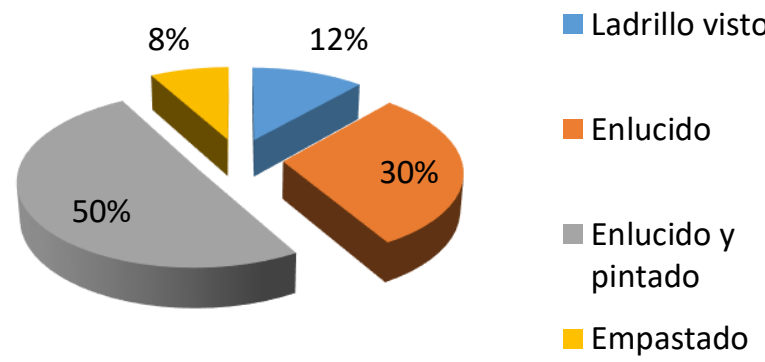

Gráfico 8. Acabado que predomina en la pared de la vivienda. Fuente: Elaboración propia 


\section{ACABADO QUE PREDOMINA EN EL PISO DE DORMITORIOS}

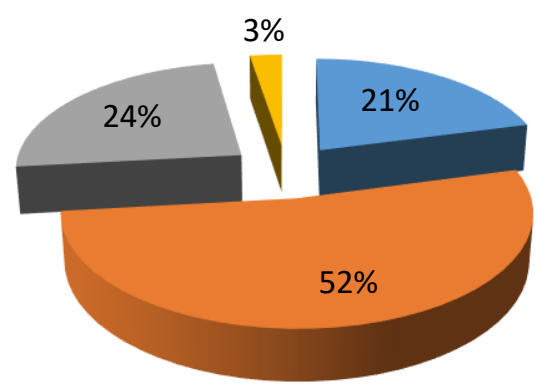

$$
\begin{aligned}
& \text { Encementado } \\
& \text { Madera } \\
& \text { Cerámica } \\
& \text { Otros }
\end{aligned}
$$

\section{Gráfico 9. Acabado que predomina en el piso de dormitorios.} Fuente: Elaboración propia

Los espacios necesarios que requieren en su vivienda permiten deducir sus hábitos de vida, en general estos espacios son: sala, comedor, cocina, tres dormitorios, un baño completo, esto se puede reflejar en el gráfico 10, donde se da menos importancia a ciertos espacios.

\section{ESPACIOS QUE CONSIDERAN MENOS NECESARIOS}

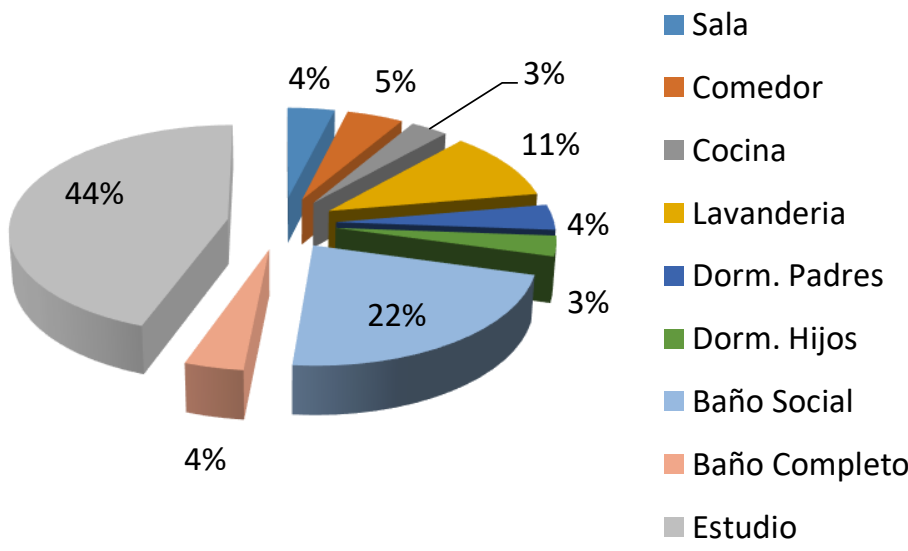

\section{Gráfico 10. Espacios que consideran menos necesarios.} Fuente: Elaboración propia

Si bien el 78\% de las familias no comparten el espacio de lavandería, el 67\% de los hogares encuestados dicen que, si aceptarían lavandería comunal a efecto de reducir costos, pero con espacio individual para el secado de ropa.

Un $82 \%$ de los encuestados dicen estar dispuestos a participar en la construcción de su edificio de vivienda, esto nos permite plantear estrategias para abaratar costos de la construcción. Ver gráfico 11. 


\section{PREDISPOSICIÓN A PARTICIPAR EN LA CONSTRUCCIÓN DE SU EDIFICIO DE VIVIENDA}

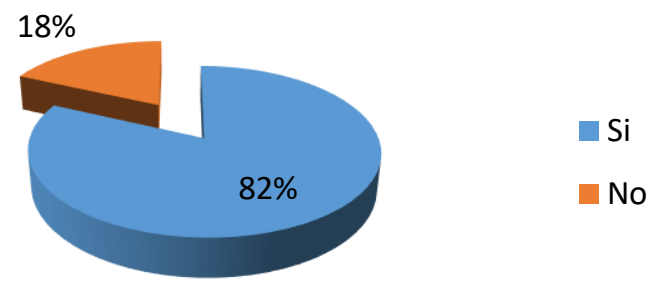

\section{Gráfico 11. Predisposición a participar en la construcción de su edificio de vivienda. \\ Fuente: Elaboración propia}

Conforme lo expuesto, con el análisis de las variables cuantitativas y cualitativas a partir de los ámbitos: Social, económico y habitacional, se plantean una serie de estrategias que pueden ser consideradas como lineamientos para el desarrollo de viviendas sociales, las cuales se exponen en las tablas a seguir:

Tabla 02. Variables y estrategias. Patrones sociales

\begin{tabular}{|c|c|c|c|}
\hline $\begin{array}{c}\text { ÁMBIT } \\
\mathbf{O}\end{array}$ & VARIABLE & RESULTADOS & ESTRATEGIAS \\
\hline \multirow{5}{*}{ SOCIAL } & $\begin{array}{l}\text { Porcentaje de } \\
\text { hijos varones que } \\
\text { trabajan }\end{array}$ & $\begin{array}{l}\text { En esta encuesta podemos indicar que el } \\
69 \% \text { de hijos varones laboran y el } 31 \% \\
\text { restante no lo hace }\end{array}$ & $\begin{array}{l}\text { El espacio de habitación del hijo debe } \\
\text { considerarse como un área de descanso, } \\
\text { aunque no está por demás prever una estación } \\
\text { puntual para trabajo/estudio remoto. }\end{array}$ \\
\hline & $\begin{array}{l}\text { Porcentaje de } \\
\text { hijas mujeres que } \\
\text { trabajan }\end{array}$ & $\begin{array}{l}\text { En esta encuesta podemos indicar que el } \\
76 \% \text { de hijas mujeres laboran y el } 24 \% \\
\text { restante no lo hace }\end{array}$ & $\begin{array}{l}\text { El espacio de habitación de la hija debe } \\
\text { considerarse como un área de descanso, } \\
\text { aunque no está por demás prever una estación } \\
\text { puntual para trabajo/estudio remoto. }\end{array}$ \\
\hline & $\begin{array}{l}\text { Personas con } \\
\text { discapacidad }\end{array}$ & $\begin{array}{l}\text { En este estudio se puede indicar el } 23 \% \\
\text { tiene discapacidad mientras que el } \\
\text { porcentaje mayoritario no lo tiene. }\end{array}$ & $\begin{array}{l}\text { Esta variable permitirá que se consideren } \\
\text { espacios con accesibilidad universal }\end{array}$ \\
\hline & $\begin{array}{l}\text { Adultos mayores } \\
\text { en la familia }\end{array}$ & $\begin{array}{l}\text { En este estudio se puede indicar el } 19 \% \\
\text { tiene personas adultas mayores, mientras } \\
\text { que el porcentaje mayoritario no lo tiene. }\end{array}$ & $\begin{array}{l}\text { Propuesta de espacios con importante nivel de } \\
\text { confort para adultos mayores. }\end{array}$ \\
\hline & $\begin{array}{l}\text { Donde dejan a sus } \\
\text { niños mientras } \\
\text { laboran } \\
\text { diariamente }\end{array}$ & $\begin{array}{l}\text { Los padres de familia tienen hijos que } \\
\text { necesita de cuidados por lo que el } 65 \% \text { los } \\
\text { dejan en casa, el } 21 \% \text { los encargan con } \\
\text { familiares, el } 9 \% \text { los dejan con otras } \\
\text { personas y el } 5 \% \text { los dejan en guarderías. }\end{array}$ & $\begin{array}{l}\text { Se propondrán ámbitos, considerando que los } \\
\text { hijos permanecen en la vivienda, por lo que } \\
\text { los espacios deberán tomar en cuenta esta } \\
\text { condición }\end{array}$ \\
\hline
\end{tabular}

Elaboración: Toledo A.

Tabla 03. Variables y estrategias. Patrones sociales

\begin{tabular}{clll}
\hline ÁMBITO & VARIABL & \multicolumn{1}{c}{ RESULTADOS } & ESTRATEGIAS \\
\hline & Edad de & De acuerdo a la información recopilada en relación al & Se planteará al menos una habitación \\
jefes de & jefe de hogar, el 54\% corresponde a personas de entre & para jefes de familia adultos \\
hogar & 40 y 50 años; el 31\% a personas de entre 30 y 40 años & \\
& y el 15\% de entre 20 y 30 años
\end{tabular}


Número de Conforme los resultados obtenidos, se evidencia que familias que el 75\% del total de encuestados, refiere que es una viven por sola familia, aquella que vive en las unidades de casa habitación, seguido de un 19\% correspondiente a dos familias viviendo en la edificación. La ocupación de tres familias corresponde a un $4 \%$ y un $2 \%$ a cuatro familias.

Edad de los Analizada la encuesta, se obtiene que la edad de los hijos hijos varones fluctúa entre 10 y 20 años (42\%). El varones $\quad 30 \%$ corresponde a hijos con edades entre 20 y 30 años; y, de 0 a 10 años, un porcentaje del $28 \%$.

Edad de hijas mujeres

Cargas familiares

Estatus laboral del padre de familia

Estatus laboral de la madre de familia

El análisis correspondiente nos da como resultado que 3 personas abarca de manera similar el $31 \%$ de la encuesta, mientras que cuando se tiene 1 carga familiar el porcentaje es de $15 \%$ y cuando el número de cargas es de 4 personas o más el porcentaje es de $23 \%$.

En este ítem se obtiene resultados que indican que el $89 \%$ de padres de familia trabaja y el $11 \%$ no lo hace.

En este ítem se obtiene resultados que indican que el $56 \%$ de padres de familia trabaja y el $44 \%$ no lo hace.
La propuesta deberá considerar una familia por cada unidad habitacional

Se hace necesaria la planificación de al menos un espacio de habitación para un hijo varón, que de acuerdo a la encuesta mantiene un rango de edad prioritaria entre 10 y 30 años (criterio de flexibilidad espacial)

Se hace necesaria la planificación de al menos un espacio de habitación para una hija mujer, que de acuerdo a la encuesta mantiene un rango de edad prioritaria entre 0 y 20 años (criterio de flexibilidad espacial)

Se requiere que la vivienda sea el número de cargas familiares en una cantidad de 2 y entre 4 y 5 personas por unidad de habitación

Se debe plantear una propuesta que considere el hecho de que el padre de familia labora fuera de casa

Se debe plantear una propuesta que considere el hecho de que un importante porcentaje de madres de familia hace su actividad en casa. Será importante el planteamiento de ámbitos cómodos para labores domésticas.

\section{Elaboración: Toledo A.}

Tabla 04. Variables y estrategias. Patrones económicos

\begin{tabular}{|c|c|c|}
\hline ÁMBITO & VARIABLE & RESULTADOS \\
\hline & $\begin{array}{l}\text { Número de miembros } \\
\text { que aportan } \\
\text { económicamente al } \\
\text { hogar }\end{array}$ & $\begin{array}{l}\text { De acuerdo a la información } \\
\text { obtenida se indica que } 2 \\
\text { personas en un porcentaje del } \\
44 \% \text { aportan económicamente al } \\
\text { hogar, al menos } 1 \text { persona se } \\
\text { tiene un porcentaje del } 36 \%, 3 \\
\text { personas de la familia, } 3 \\
\text { habitantes de la familia aporta en } \\
\text { un porcentaje del } 16 \% \text {, y más de } \\
3 \text { personas aportan en un } \\
\text { porcentaje del } 4 \% \text {. }\end{array}$ \\
\hline & $\begin{array}{l}\text { Valor de ingresos } \\
\text { económicos por familia }\end{array}$ & $\begin{array}{l}\text { De este ítem analizado, los } \\
\text { valores de ingresos económicos } \\
\text { por familia se tiene que el } 36 \% \\
\text { tiene ingresos más allá de los } \$ \\
400 \text {, el } 35 \% \text { los valores } \\
\text { obtenidos están entre los } \$ 301 \text { - } \\
\$ 400 \text {, el } 17 \% \text { tiene ingresos de } \$ \\
201 \text { - \$ } 300 \text { y el } 12 \% \text { de } \$ 100-\$ \\
200 \text {. }\end{array}$ \\
\hline
\end{tabular}

Al analizar este ítem se llega a la
conclusión de que la familia en su conjunto
está en la capacidad de hacer una inversión
(así sea mínima) para la compra o
adquisición de una vivienda social.

Si se toma en cuenta que la media del ingreso por familia es de alrededor de 400 dólares, y considerando que de ese valor un $30 \%$ podría ser destinado a una cuota mensual para vivienda, el valor debiera ser de alrededor de $\$ 120$ dólares 
ECONÓMICO

Valor de egresos económicos por familia

Monto económico de una familia para adquirir un departamento

Medio de transporte que se utiliza en el hogar
En este indicativo se tiene que

las encuestas nos dan un resultado bastante semejante de gastos o egresos en estas familias, por lo que los resultados son los siguientes: el $27 \%$ tiene egresos que van de \$ 301 a $\$ 400$, el $26 \%$ de $\$ 201$ a $\$$ 300 , el $24 \%$ tiene egresos superiores a $\$ 400$ y el $23 \%$ gastos que van de \$ 100 a \$200.

Luego del correspondiente estudio de encuesta sobre el monto económico total máximo que podría utilizar una familia para la compra de un apartamento los resultados nos indican que el $39 \%$ no podría invertir ningún valor para la obtención un una vivienda, el $29 \%$ estaría dispuesto a invertir \$20000, el 17\% invertiría \$ 25000 , el $9 \%$ lo haría con una inversión de $\$ 30000$ y el porcentaje restante que sería el $6 \%$ invertiría \$35000 o incluso algo más.

De la información recabada, el $71 \%$ de las familias ocupan el transporte público, mientras que el $28 \%$ tiene su propio medio de transporte y apenas el $1 \%$ ocupa el alquiler de transporte.
La media del gasto por familia es de alrededor de 200 dólares
De acuerdo a lo indicado, se podría considerar como inversión para unidad de vivienda un monto de alrededor de $\$ 20000$ dólares. En este punto se podría llegar a un acuerdo con los diferentes ministerios del estado o con los gobiernos autónomos descentralizados para un plan de vivienda para familias que no tienen patrimonio económico, pero es lógico que debería existir una contraparte que a lo mejor sería emplazar dicha vivienda en un terreno familiar que cumpla con las condiciones necesarias

Con los parámetros obtenidos en los resultados se sugiera que por cada 3 viviendas o predio familiar se disponga de una plaza de parqueamiento, de preferencia se propondrán espacios de parqueo comunitario.

\section{Elaboración: Toledo A.}

Tabla 05. Variables y estrategias. Patrones habitacionales y constructivos

\begin{tabular}{|c|c|c|}
\hline ÁMBITO & VARIABLE & RESULTADOS \\
\hline & $\begin{array}{l}\text { La vivienda es } \\
\text { propia o arrendada? }\end{array}$ & $\begin{array}{l}\text { En esta consulta se tiene que el } 65 \% \\
\text { de familias disponen de una viviend } \\
\text { propia, y el restante en una vivienda } \\
\text { arrendada. }\end{array}$ \\
\hline & $\begin{array}{l}\text { Acabado } \\
\text { predominante en la } \\
\text { pared de la vivienda. }\end{array}$ & $\begin{array}{l}\text { En este indicativo se tiene que el } \\
\text { enlucido y pintado de las paredes } \\
\text { abarca en un } 50 \% \text { de la muestra } \\
\text { analizada, el } 30 \% \text { tiene solo las } \\
\text { paredes pintadas, el } 12 \% \text { la } \\
\text { mampostería es de ladrillo visto y el } \\
8 \% \text { es empastado. }\end{array}$ \\
\hline \multirow[t]{2}{*}{$\begin{array}{l}\text { HABITACIONAL } \\
\text { Y } \\
\text { CONSTRUCTIVO }\end{array}$} & $\begin{array}{l}\text { Tipo de piso que } \\
\text { predomina en los } \\
\text { dormitorios }\end{array}$ & $\begin{array}{l}\text { El porcentaje predominante en este } \\
\text { estudio son los pisos de madera en } \\
\text { un } 52 \% \text {, el } 24 \% \text { son pisos de } \\
\text { cerámicas, el } 21 \% \text { pisos solo } \\
\text { encementados y el } 3 \% \text { tiene otro } \\
\text { acabado en los pisos. }\end{array}$ \\
\hline & $\begin{array}{l}\text { Disponen de baño } \\
\text { social }\end{array}$ & $\begin{array}{l}\text { En este estudio se obtiene que el } \\
56 \% \text { no dispone de un baño social, } \\
\text { mientras que el } 44 \% \text { si dispone. }\end{array}$ \\
\hline
\end{tabular}

Conforme los resultados, para la propuesta se considerarán paredes enlucidas y pintadas.

De acuerdo a lo observado, se evidencia que los pisos deberán ser maderados y con revestimiento cerámico, dependiendo de los ambientes específicos.

Se debería considerar al menos 1 baño social, que servirá para un, mejor estilo de vida a los habitantes. 
Disponen de baño completo

La actividad de reunión familiar es
En este estudio se obtiene que el $90 \%$ dispone de un baño social, mientras que el $10 \%$ no dispone.

El lugar predominante para reuniones de familia se lo hace en la sala teniendo este un porcentaje del $41 \%$, el $31 \%$ en el comedor, el $16 \%$ en la cocina, el $9 \%$ en el comedor y apenas el $3 \%$ en el cuarto
Se debería considerar al menos 1 baño completo, ya que por cuestión de sanidad, salud esto mejora sustancialmente la vida de los habitantes de las viviendas

En la búsqueda de optimización de espacios se plantea que los ambientes sociales sean abiertos, sala-comedorcocina

\section{Elaboración: Toledo A.}

Tabla 06. Variables y estrategias. Patrones habitacionales y constructivos

\begin{tabular}{|c|c|c|c|}
\hline ÁMBITO & VARIABLE & RESULTADOS & ESTRATEGIAS \\
\hline & $\begin{array}{l}\text { Comparten el espacio } \\
\text { de lavandería con otras } \\
\text { familias }\end{array}$ & $\begin{array}{l}\text { En este análisis se tiene que el } \\
78 \% \text { no comparte lavandería, } \\
\text { mientras que el } 22 \% \text { si lo hace }\end{array}$ & $\begin{array}{l}\text { Lo más recomendable es que cada } \\
\text { vivienda tenga su espacio de lavado y un } \\
\text { área de secado de ropa a cielo abierto o } \\
\text { semicubierto }\end{array}$ \\
\hline & $\begin{array}{l}\text { Aceptarían una } \\
\text { lavandería comunal }\end{array}$ & $\begin{array}{l}\text { Este estudio demuestra el el } 67 \% \\
\text { de personas aceptaría compartir } \\
\text { una lavandería comunal, } \\
\text { mientras que el } 33 \% \text { restante no. }\end{array}$ & $\begin{array}{l}\text { Dependiendo de la necesidad y el área por } \\
\text { cada unidad de vivienda, se podrían } \\
\text { pensar en espacios de lavandería de uso } \\
\text { comunitario. }\end{array}$ \\
\hline \multirow[t]{3}{*}{$\begin{array}{l}\text { HABITACIONA } \\
\text { L Y } \\
\text { CONSTRUCTIV } \\
\text { O }\end{array}$} & $\begin{array}{l}\text { Espacios que } \\
\text { consideran menos } \\
\text { necesarios en las } \\
\text { viviendas }\end{array}$ & $\begin{array}{l}\text { Los habitantes de las viviendas } \\
\text { consideran en un } 44 \% \text { que la } \\
\text { parte menos importante de la } \\
\text { casa es el estudio, seguido por } \\
\text { un } 22 \% \text { que considera que el } \\
\text { lugar menos importante es un } \\
\text { baño social, el 11\% considera no } \\
\text { importante a la lavandería, } \\
\text { mientras que en un porcentaje } \\
\text { del } 4 \% \text { tanto el dormitorio de } \\
\text { padres como el baño completo } \\
\text { es menos importante, el } 5 \% \\
\text { piensa que la cocina es el lugar } \\
\text { menos importante de la casa, y } \\
\text { por último en un porcentaje del } \\
3 \% \text { se indica que el dormitorio } \\
\text { de hijos y la cocina es la parte } \\
\text { menos importante de la } \\
\text { vivienda. }\end{array}$ & $\begin{array}{l}\text { De acuerdo a lo indicado, se podría } \\
\text { optimizar el espacio de trabajo en cada } \\
\text { una de las habitaciones de la vivienda. }\end{array}$ \\
\hline & $\begin{array}{l}\text { Predisposición a } \\
\text { participar en la } \\
\text { construcción del su } \\
\text { edificio de vivienda. }\end{array}$ & $\begin{array}{l}\text { El } 82 \% \text { está de acuerdo en } \\
\text { aportar para la construcción de } \\
\text { su vivienda, mientras que la } \\
\text { parte restante no está de } \\
\text { acuerdo. }\end{array}$ & $\begin{array}{l}\text { Se hace necesario plantear alternativas de } \\
\text { sistemas de autoconstrucción en vivienda, } \\
\text { asumiendo la predisposición de los } \\
\text { usuarios. }\end{array}$ \\
\hline & $\begin{array}{l}\text { Número de baños } \\
\text { completos que } \\
\text { requieren las familias }\end{array}$ & $\begin{array}{l}\text { En esta análisis se demuestra } \\
\text { que el } 56 \% \text { de las familias } \\
\text { desean tener } 2 \text { baños completos } \\
\text { en su vivienda, el } 29 \% \text { desea } \\
\text { solo } 1 \text { baño completo y el } 15 \% \\
\text { quisiera tener } 2 \text { baños o más. }\end{array}$ & $\begin{array}{l}\text { De acuerdo a lo indicado, sería pertinente } \\
\text { plantear la unidad de vivienda con un } \\
\text { baño social ( } 1 / 2 \text { baño) y un baño completo }\end{array}$ \\
\hline
\end{tabular}

Elaboración: Toledo A. 


\section{Conclusiones}

- La vivienda, ya sea como capital tangible, activos o méritos, debe utilizarse para garantizar las necesidades de vida de los diferentes hogares a todo nivel. Asimismo, la condición de un activo excede su alcance físico (construcción) e involucra todos los aspectos de la determinación de su entorno y ubicación relativa. En este sentido, cuando se ha superado el acceso a tener una vivienda y la oportunidad de ingresar a la ciudad, se ha convertido en un aspecto básico para eliminar la pobreza familiar.

- Las personas de estrato social bajo que no tienen disponibilidad de acceder a una vivienda, cuando no acuden a un alquiler en condiciones inestables (o después de hacerlo), procederán a encontrar su estabilidad en invasiones y asentamientos irregulares apropiándose de manera ilegal de terrenos y lotes para ahí emplazar sus casas y así alcanzar a tener "algo propio" y conformar su patrimonio familiar; no hay duda de que esa es la más adecuada lógica y necesidad de criterio de las personas más pobres, y las políticas públicas de vivienda no pueden (o no quieren) tomar medidas efectivas para brindar a las personas opciones alternativas.

- El estudio realizado, analizó tres secciones importantes para determinar el comportamiento de las familias de estrato social, que servirán para la implementación de vivienda social de acuerdo a sus necesidades prioritaria. Con ello, se determina que los mayores requerimientos y sugerencias son:

- El resultado refiere que hay un porcentaje importante de dos o más familias habitando en una sola unidad de vivienda, por lo que lo más conveniente sería una casa para cada familia. Se recomienda que, en estas viviendas, la mampostería utilizada sea de ladrillo o bloque, enlucida y pintada, con pisos de madera y revestimiento cerámico que dependerá de los ambientes específicos; lógicamente, se debe analizar el factor económico para la adquisición y calidad de los materiales, y que los mismos permitan un adecuado nivel de mantenimiento, tanto en costo como en tiempo de ejecución.

- La unidad de habitación social debería ser planificada para un número de entre 4 y 5 habitantes, optimizando los espacios con ambientes sociales abiertos, salacomedor-cocina; siendo estos también cómodos para labores domésticas, ya que existe un porcentaje considerable de madres de familia que realiza actividades en casa. Se debe considerar una habitación para padres o jefes de familia, al igual que una habitación individual para hijo e hija, espacio que debería preverse como un área de descanso, que dispondrá de una estación puntual para trabajo o estudio remoto. Adicionalmente, los espacios serán concebidos tomando en cuenta el criterio de accesibilidad universal, debido al importante porcentaje de personas con capacidades diferentes y adultos mayores; por ello, se deberían implementar espacios útiles y confortables para este tipo de personas.

- La casa, deberá disponer de al menos un baño social y uno completo, área de lavado y secado de ropa, preferiblemente al aire libre o un patio semi-cubierto para estos menesteres. Ahora, para una mejor optimización de recursos y espacio 
dependiendo de la necesidad de cada hogar, se puede plantear el emplazamiento de un área comunal para el uso de un grupo de familias. Al haber usuarios menores (infantes) que se quedan en casa mientras sus padres trabajan, se planificarán espacios para dar seguridad a los niños; o, de ser el caso, se podría proponer una guardería en el conjunto residencial para el cuidado de los pequeños.

- Analizando la parte económica de las familias se indica un importante porcentaje de los miembros de familia tienen actividad laboral definida. De ello se deriva que la media de ingresos de todos los habitantes del hogar está en alrededor de 400 dólares y la media de gasto familiar bordea los 200 dólares. Con ello, el núcleo familiar en su conjunto estaría en capacidad de hacer una inversión de un monto que bordearía los 20000 dólares destinados a la adquisición de una vivienda y para las personas que no tienen un patrimonio económico, considerar acuerdos con entes públicos o privados para acceder a un plan de vivienda, o la posibilidad de inversión de esos recursos en un predio propio que disponga de servicios adecuados para el proceso constructivo y de edificación, empleando sistemas constructivos actuales en los que prime la autoconstrucción, esto gracias a la predisposición de los interesados y la imperativa necesidad de acceso a una vivienda social digna.

AGRADECIMIENTOS: El presente artículo es parte del trabajo de investigación y titulación del Programa de Maestría en Construcción con Mención en Administración de la Construcción Sustentable de la Universidad Católica de Cuenca, por ello agradecemos a todos y cada uno de los instructores por los conocimientos e información brindados para la elaboración del trabajo.

\section{Referencias bibliográficas}

Estudio del BID: América Latina y el Caribe encaran creciente déficit de vivienda |IADB. (n.d.). Retrieved February 1, 2018, from https://www.iadb.org/es/noticias/comunicados-de-prensa/2012-0514/deficit-devivienda-en-america-latina-y-el-caribe\%2C9978.html\#getNews(9969,'”\#

Franco, P. (2013). Políticas alternativas de vivienda en América Latina y el Caribe, 1118.

Held, G. (2000). Políticas de viviendas de interés social orientadas al mercado: experiencias recientes con subsidios a la demanda en Chile, Costa Rica y Colombia. Naciones Unidas, CEPAL, Unidad de Financiamiento para el desarrollo, Div. de Comercio Internacional y Financiamiento para el desarrollo. Retrieved from https://www.cepal.org/es/publicaciones/5304-políticas-viviendas interés-social-orientadas-al-mercado-experiencias-recientes.

Inmobiliario, M. (2005). Bolet ' 1 n CF + S 29 / 30. Junio 2005. 
Muñoz, O., Patricia, P., Ochoa, R., \& Maritza, J. (2011). Estudio de Factibilidad Financiera para la Construcción y Comercialización de casas, Ubicadas en el sector de Challuabamba en la ciudad de Cuenca Estudio de Factibilidad Financiera para la construcción y, 111. Retrieved from http://www.google.com.ec/url?sa=t\&rct=j\&q=\&esrc=s\&source=web\&cd=5\&ca $\mathrm{d}=\mathrm{rja} \& u a c t=8 \&$ ved=0CEMQFjAE\&url=http://dspace.ups.edu.ec/bitstream/1234 $56789 / 1294 / 14 /$ UPS-

CT002241.pdf\&ei=QDRdU4OmN8ThsAStzIGwCA\&usg=AFQjCNGTHZxGE A7Du4Arh96knIL4qruFaQ\&bvm=bv.65397613,d.cWc

Tierra, C. C. O. N., Calle, M. B. A., Aguilar, P. J. A., Pesántez, J. F. P., \& Angélica, M. (2017). INCIDENCIA DE LA TIERRA EN EL CONFORT DE PERSONAS INVIDENTES EN AMBIENTES DE ENSEÑANZA - APRENDIZAJE.

Sustentable, A., \& La, E. N. (2007). ARQUITECTURA TRADICIONAL DE CUENCA, RECUPERACIÓN Y, (1557), 48-70

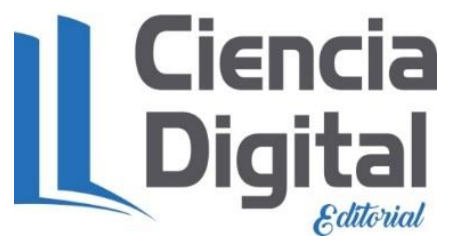




\section{PARA CITAR EL ARTÍCULO INDEXADO.}

Toledo Toledo, Álvaro, Toledo Toledo, J. F., \& Avila Calle, M. (2021). Estrategias para diseño de vivienda social en Cuenca - Ecuador, mediante un análisis cuantitativo de patrones sociales, económicos, habitacionales y constructivos. Ciencia Digital, 5(2), 7999. https://doi.org/10.33262/cienciadigital.v5i2.1595

\section{Ciencia \\ LDigital}

El artículo que se publica es de exclusiva responsabilidad de los autores y no necesariamente reflejan el pensamiento de la Revista Ciencia Digital.

El artículo queda en propiedad de la revista y, por tanto, su publicación parcial y/o total en otro medio tiene que ser autorizado por el director de la Revista Ciencia Digital.
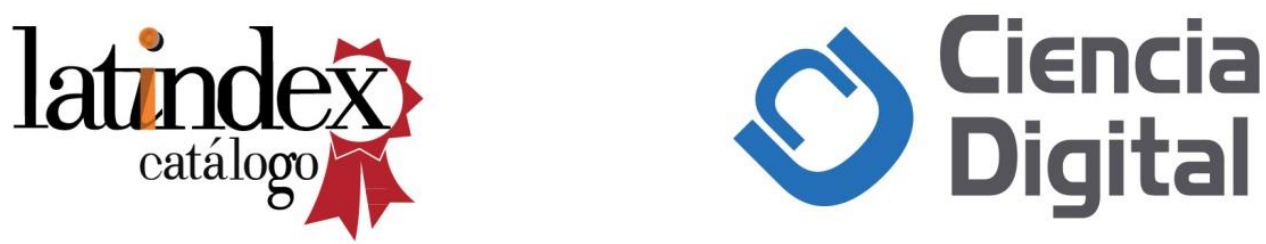